Proceedings of the Edinburgh Mathematical Society (2008) 51, 27-44 (C)

DOI:10.1017/S0013091505001483 Printed in the United Kingdom

\title{
LOOP HOMOLOGY AS FIBREWISE HOMOLOGY
}

\author{
M. C. CRABB \\ Department of Mathematical Sciences, University of Aberdeen, \\ Aberdeen AB24 3UE, UK (m.crabb@maths.abdn.ac.uk)
}

(Received 1 November 2005)

\begin{abstract}
The loop homology ring of an oriented closed manifold, defined by Chas and Sullivan, is interpreted as a fibrewise homology Pontrjagin ring. The basic structure, particularly the commutativity of the loop multiplication and the homotopy invariance, is explained from the viewpoint of the fibrewise theory, and the definition is extended to arbitrary compact manifolds.
\end{abstract}

Keywords: fibrewise homology; Chas-Sullivan loop homology; Pontrjagin ring

2000 Mathematics subject classification: Primary 55R70; 55P43

\section{Introduction}

In [3] Chas and Sullivan defined the loop product on the (rational) homology, $H_{*+d}(\mathcal{L} M)$, of the free loop space of an oriented closed $d$-manifold $M$, with a dimension shift $d$, and showed that the product gave $H_{*+d}(\mathcal{L} M)$ the structure of a (graded) commutative associative $H^{*}(M)$-algebra. The free loop space $\mathcal{L} M$, defined as the space of continuous loops $\alpha: \mathbb{R} / \mathbb{Z} \rightarrow M$, fibres over $M$ by evaluation at $0: \alpha \in \mathcal{L} M \mapsto \alpha(0) \in M$, and the fibre at $x \in M$ is naturally identified with the space of based loops $\Omega(M, x)$ in the space $M$ with basepoint $x$. In this framework the loop product can be interpreted as a fibrewise Pontrjagin product in the fibrewise homology of $p: \mathcal{L} M \rightarrow M$ over $M$. This paper gives an account of loop homology from this point of view, with some examples. Although the elementary definitions can be made for any compact Euclidean neighbourhood retract $M$, the proof of the commutativity of the loop product given in $\S 5$ requires $M$ to be a smooth, but not necessarily orientable, closed manifold. The results are extended to a compact manifold with non-empty boundary $\partial M$ by looking at the relative fibrewise homology over $(M, \partial M)$.

Granted some familiarity with fibrewise homology theory, the definition of loop homology as a fibrewise theory and the verification of its basic properties are straightforward exercises. In $\S 2$ we provide a brief review of fibrewise homology theory. For more details the reader is referred to $[\mathbf{9}$, Part II, $\S 15]$; the account of fibrewise stable homotopy given there was written primarily as a source for applications to geometry, and this is one such application (see also [8]). Section 3 describes the basic definition and structure of loop homology as a fibrewise homology theory. In $\S 4$ we relate the construction to the 
definition given by Cohen and Jones [4] in terms of the Thom space of the pull-back $-p^{*} \tau M$ to $\mathcal{L} M$ of the negative of the tangent bundle $\tau M$ of the smooth manifold. (The relationship between the two definitions is, in fact, a rather old result, going back at least to [10].) Section 5 treats the commutativity of the loop product.

There is now a fairly extensive literature on loop homology. The reader is referred, in particular, to [12-16].

\section{A review of fibrewise homology theory}

We fix a base space $B$, which will be a compact Euclidean neighbourhood retract (ENR). All fibrewise pointed spaces over $B$ will be understood to be locally fibre homotopy trivial with each fibre of the homotopy type of a pointed CW complex. We also make the technical restriction that fibrewise pointed spaces be homotopy well pointed (as in [9]). A subscript $x$ is used to indicate the fibre of a fibrewise space at a point $x \in B$ of the base.

\subsection{Definitions}

Let $E \rightarrow B$ and $F \rightarrow B$ be fibrewise pointed spaces with the fibres of $E$ being of the homotopy type of finite complexes. Denoting the Eilenberg-MacLane space $K(\mathbb{Z}, n)$ by $K_{n}$, we define the fibrewise cohomology groups (with $\mathbb{Z}$-coefficients) for $i \in \mathbb{Z}$ as direct limits of sets of fibrewise pointed homotopy classes over $B$ :

$$
H_{B}^{i}\{E ; F\}:=\underset{n}{\lim }\left[\Sigma_{B}^{n} E ;\left(B \times K_{n+i}\right) \wedge_{B} F\right]_{B},
$$

where $\Sigma_{B}$ is the fibrewise suspension. More generally, if $A \subseteq B$ is a closed sub-ENR of $B$, we define the relative groups

$$
H_{(B, A)}^{i}\{E ; F\}:=\underset{n}{\lim }\left[\sum_{B}^{n} E ;\left(B \times K_{n+i}\right) \wedge_{B} F\right]_{(B, A)}
$$

as homotopy classes of fibrewise maps over $B$ which are null (that is, zero) over $A$.

If $B$ is a point, we drop the suffix and write simply $H^{*}\{X ; Y\}$ for pointed CW complexes $X$ and $Y$ (with $X$ finite). (The notation is not widely used in algebraic topology, but the cognate $K K$-notation for Kasparov's $K$-groups of $C^{*}$-algebras is standard.) Thus, $H^{i}\left\{X ; S^{0}\right\}$ is the (reduced) cohomology $\tilde{H}^{i}(X)$ of the pointed space $X$, and $H^{i}\left\{S^{0} ; Y\right\}=\tilde{H}_{-i}(Y)$ is the homology of $Y$. In simplicial terms, $H^{i}\{X ; Y\}$ is the $\mathbb{Z}$-module of chain homotopy classes of chain maps of degree $i$ from the reduced singular chain complex $\tilde{C}_{*}(X)$ of $X$ to $\tilde{C}_{*}(Y)$.

In general, we refer to $H_{B}^{*}\left\{E ; B \times S^{0}\right\}$ as the fibrewise cohomology of the fibrewise pointed space $E \rightarrow B$, and to $H_{B}^{*}\left\{B \times S^{0} ; F\right\}$ as the fibrewise homology of $F$ over $B$.

\subsection{Fibrewise cohomology}

The fibrewise cohomology group is easily seen to be just the (reduced) cohomology group of $E$ modulo the subspace $B$ included as the fibrewise basepoint:

$$
H_{B}^{i}\left\{E ; B \times S^{0}\right\}=\tilde{H}^{i}(E / B) .
$$


More generally, when $F$ is trivial, say $F=B \times Y$, we have the identification:

$$
H_{B}^{i}\{E ; B \times Y\}=H^{i}\{E / B ; Y\} .
$$

\subsection{Fibrewise homology}

The fibrewise homology groups do not have a corresponding classical interpretation. We shall be concerned in this paper with the functor

$$
X \mapsto H_{B}^{*}\{B \times X ; F\}
$$

on pointed finite complexes $X$, for a fixed fibrewise space $F \rightarrow B$. In the special case that $B$ is a closed manifold there is a representability theorem, stated precisely as Proposition 4.1:

$$
H_{B}^{*}\{B \times X ; F\}=H^{*}\left\{X ;\left(N \wedge_{B} F\right) / B\right\},
$$

where $N$ is a certain stable space over $B$ (an appropriate fibrewise desuspension of the fibrewise one-point compactification of the normal bundle of an embedding of $B$ in some Euclidean space).

\subsection{Products}

The fibrewise cohomology has smash and composition products:

$$
\begin{aligned}
\wedge: & H_{B}^{i}\{E ; F\} \otimes H_{B}^{i^{\prime}}\left\{E^{\prime} ; F^{\prime}\right\} \rightarrow H_{B}^{i+i^{\prime}}\left\{E \wedge_{B} E^{\prime} ; F \wedge_{B} F^{\prime}\right\}, \\
\circ: & H_{B}^{j}\{E ; F\} \otimes H_{B}^{i}\{D ; E\} \rightarrow H_{B}^{j+i}\{D ; F\}
\end{aligned}
$$

(for fibrewise pointed spaces $E, E^{\prime}, F, F^{\prime}$ and $D$ over $B$ with the fibres of $E, E^{\prime}$ and $D$ finite). In particular, $H_{B}^{*}\{E ; F\}$ is a (graded) $H^{*}(B)$-module.

\subsection{The Serre spectral sequence}

The classical construction generalizes to give a Serre spectral sequence, functorial for $E$ and $F$ in the category of fibrewise pointed spaces, with $E_{2}$-term

$$
E_{2}^{p, q}=H^{p}\left(B ; \mathcal{H}^{q}\right)
$$

where $\mathcal{H}^{q}$ is the local coefficient system with $\mathcal{H}_{x}^{q}=H^{q}\left\{E_{x} ; F_{x}\right\}, x \in B$, converging to $H_{B}^{*}\{E ; F\}$. The differential $\mathrm{d}^{r}$ has degree $(r,-r+1)$ (see [9, Part II, Proposition 15.20]). The spectral sequence is multiplicative, that is, the differentials are derivations, with respect to the smash and composition products (in $\S 2.4$ ).

\subsection{The fibrewise Pontjragin product}

Let $\Phi \rightarrow B$ be a locally fibre homotopy trivial fibrewise space over $B$ (with fibres of the homotopy type of CW complexes). We write $\Phi_{+B} \rightarrow B$ for the fibrewise pointed space obtained by adjoining a disjoint basepoint in each fibre. (Thus, the total space $\Phi_{+B}$ is the disjoint union of $\Phi$ and $B$.) 
Suppose that $\Phi \rightarrow B$ is a fibrewise Hopf space with (homotopy) associative multiplication $m: \Phi \times{ }_{B} \Phi \rightarrow \Phi$ and (homotopy) identity $e: B \rightarrow \Phi$. Then we have an associative Pontrjagin product

$$
m_{*}: H_{B}^{*}\left\{E ; \Phi_{+B}\right\} \otimes H_{B}^{*}\left\{E^{\prime} ; \Phi_{+B}\right\} \rightarrow H_{B}^{*}\left\{E \wedge_{B} E^{\prime} ; \Phi_{+B}\right\} .
$$

In particular, $H_{B}^{*}\left\{B \times S^{0} ; \Phi_{+B}\right\}$ is an associative algebra over $H^{*}(B)$ with identity element $e_{*} \in H_{B}^{0}\left\{B \times S^{0} ; \Phi_{+B}\right\}$.

\section{The definition of loop homology}

Let $M$ be a compact ENR, for example, a closed manifold or finite complex. Consider the fibrewise pointed space $M \times M \rightarrow M:(x, y) \mapsto x$, pointed by the diagonal section: $x \mapsto(x, x)$. We shall show that it is a pointed homotopy fibre bundle, in the terminology of [9], that is, it is locally fibre homotopy trivial as a fibrewise pointed space. (In more standard, but less geometric, terminology, $M \times M \rightarrow M$ is a based fibration.)

Lemma 3.1. Let $M$ be a compact ENR. Then the fibrewise pointed space $M \times M \rightarrow$ $M$, pointed by the diagonal section, is locally fibre homotopy trivial.

Proof. We may assume that $M$ is a subspace of an open subspace $U$ of some Euclidean space $V$ and that $r: U \rightarrow M$ is a retraction. Fix $a \in M$. There is a neighbourhood $W$ of $a$ in $M$ such that $x \pm(b-a) \in U$ for all $x \in M, b \in W$. Define $\phi, \psi: W \times M \rightarrow W \times M$ by $\phi(b, x)=(x, f(b, x))$, where $f(b, x)=r(x+(b-a))$, and $\psi(b, x)=(b, g(b, x))$, where $g(b, x)=r(x-(b-a))$. Thus, $\phi(b, a)=(b, b)$ and $\psi(b, b)=(b, a)$. By an appropriate choice of $W$ we may arrange that $h_{t}(b, x)=(1-t) f(b, g(b, x))+t x \in U$ and $k_{t}(b, x)=$ $(1-t) g(b, f(b, x))+t x \in U$ for all $x \in M, b \in W, t \in[0,1]$. Notice that $h_{t}(b, b)=b$ and $k_{t}(b, a)=a$. Then $r \circ h_{t}$ and $r \circ k_{t}$ give homotopies $\phi \circ \psi \simeq 1$ and $\psi \circ \phi \simeq 1$. The map $\phi$ is, thus, a local fibre homotopy trivialization of the fibrewise pointed space $M \times M \rightarrow M$.

Remark 3.2. If $M$ is a closed manifold, the fibrewise pointed space $M \times M \rightarrow M$ is even topologically locally trivial, essentially because the diffeomorphism group of a connected closed manifold acts transitively (see, for example, [9, Part II, Example 1.20]).

The fibre of $M \times M \rightarrow M$ over $x \in M$ is the pointed space $\{x\} \times M$ with basepoint $(x, x)$. It is naturally identified, by projection, with the space $M$ with the basepoint $x$, and we shall think of the fibres in this way.

The (continuous) free loop space $\mathcal{L} M=\operatorname{map}(\mathbb{R} / \mathbb{Z}, M)$ fibres over $M$ by evaluation at the basepoint $0 \in \mathbb{R} / \mathbb{Z}$ as the fibrewise loop space $\Omega_{M}(M \times M)$ :

$$
p: \mathcal{L} M=\Omega_{M}(M \times M) \rightarrow M, \quad \alpha \mapsto \alpha(0) .
$$

The fibre at $x \in M$ is (naturally identified with) the loop space $\Omega(M, x)$ of $M$ with $x$ as basepoint. Topologically, $\mathcal{L} M$ is an absolute neighbourhood retract (ANR); for, if $M$ is a retract of an open subset of a finite-dimensional $\mathbb{R}$-vector space $V$, then $\mathcal{L} M$ is a retract of an open subset of the Banach space of continuous loops in $V$. Recall that $(\mathcal{L} M)_{+M}=\mathcal{L} M \sqcup M$ is the fibrewise pointed space over $M$ obtained by adjoining a basepoint to each fibre. 
Definition 3.3. To the compact ENR $M$ we associate the generalized cohomology theory $\mathbb{H}_{M}^{*}$ defined by

$$
\tilde{\mathbb{H}}_{M}^{*}(X)=H_{M}^{*}\left\{M \times X ;(\mathcal{L} M)_{+M}\right\}
$$

for a finite pointed complex $X$. The graded abelian group $\tilde{\mathbb{H}}_{M}^{*}(X)$ is a module over $H^{*}(M)$. As usual, for a finite complex $P$ we write $\mathbb{H}_{M}^{*}(P)=\tilde{\mathbb{H}}_{M}^{*}\left(P_{+}\right)$, where $P_{+}$is the pointed space obtained by adding a disjoint basepoint to $P$, and, if $Q$ is a subcomplex of $P$, we set $\mathbb{H}_{M}^{*}(P, Q)=\tilde{\mathbb{H}}_{M}^{*}(P / Q)$.

There is a fibrewise Hopf structure

$$
m: \Omega_{M}(M \times M) \times_{M} \Omega_{M}(M \times M) \rightarrow \Omega_{M}(M \times M)
$$

defined in the fibre over $x$ by the standard homotopy associative loop multiplication $\Omega(M, x) \times \Omega(M, x) \rightarrow \Omega(M, x)$, with (homotopy) identity

$$
e: M \rightarrow \Omega_{M}(M \times M)
$$

given in the fibre by the inclusion of the constant loop at $x$.

The fibrewise Pontrjagin multiplication (see $\S 2.6$ ) thus gives an associative loop product

$$
\mu: \tilde{\mathbb{H}}_{M}^{i}(X) \otimes \tilde{\mathbb{H}}_{M}^{j}(Y) \rightarrow \tilde{\mathbb{H}}_{M}^{i+j}(X \wedge Y) .
$$

In particular, the coefficient ring $\mathbb{H}_{M}^{*}(*)$, which (following $[\mathbf{3}, \mathbf{4}]$ ) will be called the loop homology of $M$, is an associative graded $H^{*}(M)$-algebra with identity $1 \in \mathbb{H}_{M}^{0}(*)$ given by the section $e$. (The algebra is also equipped with an involution, given by reversing loops: $\Omega_{M}(M \times M) \rightarrow \Omega_{M}(M \times M)$.)

We record two immediate consequences of the definition.

Proposition 3.4. For each point $x \in M$, there is a natural multiplicative transformation:

$$
\tilde{\mathbb{H}}_{M}^{*}(X) \rightarrow H^{*}\left\{X ;(\Omega(M, x))_{+}\right\} .
$$

Proof. This is given by restriction to fibres at $x$ :

$$
\tilde{\mathbb{H}}_{M}^{*}(X)=H_{M}^{*}\left\{M \times X ;(\mathcal{L} M)_{+M}\right\} \rightarrow H^{*}\left\{X ;(\Omega(M, x))_{+}\right\} .
$$

Proposition 3.5. There exists a natural multiplicative transformation of $H^{*}(M)$ modules:

$$
\tilde{\mathbb{H}}_{M}^{*}(X) \rightarrow \tilde{H}^{*}\left(M_{+} \wedge X\right) .
$$

Proof. The transformation is induced by the projection $\mathcal{L} M \rightarrow M$ :

$$
\tilde{\mathbb{H}}_{M}^{*}(X)=H_{M}^{*}\left\{M \times X ;(\mathcal{L} M)_{+M}\right\} \rightarrow H_{M}^{*}\left\{M \times X ; M \times S^{0}\right\}=\tilde{H}^{*}\left(M_{+} \wedge X\right) .
$$

It is evidently multiplicative with respect to $\mu$ and the cup product:

$$
\tilde{H}^{*}\left(M_{+} \wedge X\right) \otimes \tilde{H}^{*}\left(M_{+} \wedge Y\right) \rightarrow \tilde{H}^{*}\left(M_{+} \wedge(X \wedge Y)\right) .
$$


Next we investigate the dependence of the loop homology on $M$.

Lemma 3.6. Let $f: M \rightarrow N$ be a homotopy equivalence between compact ENRs. Then the fibrewise map

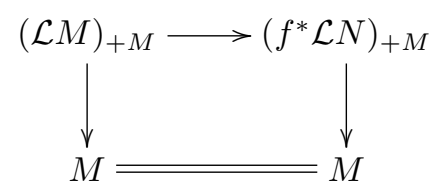

determined by $f$ is a pointed fibre homotopy equivalence.

Proof. On the fibres at $x \in M$ we have the induced map

$$
\Omega(M, x) \rightarrow \Omega(N, f(x)),
$$

which is a pointed homotopy equivalence because the ENRs $M$ and $N$ are well pointed by any choice of basepoint. In view of Dold's theorem (as, for example, in [9, Part II, Theorem 1.29]), we recognize a pointed fibre homotopy equivalence over $M$.

We thus obtain isomorphisms

$$
H_{M}^{*}\left\{M \times X ;(\mathcal{L} M)_{+M}\right\} \stackrel{f_{*}}{\cong} H_{M}^{*}\left\{M \times X ;\left(f^{*} \mathcal{L} N\right)_{+M}\right\} \stackrel{f^{*}}{\cong} H_{N}^{*}\left\{N \times X ;(\mathcal{L} N)_{+N}\right\},
$$

the first induced by the fibre homotopy equivalence in Lemma 3.6 and the second by lifting from $N$ to $M$. Denoting the composition $\left(f^{*}\right)^{-1} f_{*}$ by $f_{\#}$, we have established the following homotopy invariance property of loop homology.

Theorem 3.7 (homotopy invariance of loop homology). Let $f: M \rightarrow N$ be a homotopy equivalence between compact ENRs. Then there is an induced equivalence of multiplicative cohomology theories

$$
f_{\#}: \tilde{\mathbb{H}}_{M}^{*}(X) \rightarrow \tilde{\mathbb{H}}_{N}^{*}(X) .
$$

The Serre spectral sequence in $\S 2.5$ yields the following spectral sequence for loop homology, which has appeared, as an independent construction, in [5].

Proposition 3.8 (Serre spectral sequence for loop homology). There is a multiplicative spectral sequence with $E_{2}$-term $H^{*}\left(M ; \mathcal{H}^{*}\right)$, where $\mathcal{H}^{*}$ is the local coefficient system with $\mathcal{H}_{x}^{*}=H_{-*}(\Omega(M, x))$ at $x \in M$, converging to the loop homology ring $\mathbb{H}_{M}^{*}(*)$.

Example 3.9. The calculation of the loop homology ring $\mathbb{H}_{M}^{*}(*)$ of an odd-dimensional sphere $M=S^{2 n+1}, n \geqslant 1$, was made in [9] (see Part II, Proposition 15.28) and the discussion following Part II, Remark 15.31)) and in [5]. If we write $M$ as the unit sphere $S\left(\mathbb{C}^{n+1}\right)$ in $\mathbb{C}^{n+1}$, we can split the tangent bundle as $\tau M=\mathbb{R} \oplus \zeta$, where the fibre $\zeta_{x}$ at $x$ is the orthogonal complement of $\mathbb{C} x$ in $\mathbb{C}^{n}$. Then $\Omega(M, x)$ can be written as $\Omega S\left(\mathbb{C} x \oplus \zeta_{x}\right)$ 
and identified with $\Omega \Sigma \zeta_{x}^{+}$, where the sphere $\zeta_{x}^{+}$is the one-point compactification of $\zeta_{x}$. This allows us to write $\mathcal{L} M$ as the fibrewise loop space

$$
\mathcal{L} M=\Omega_{M} \Sigma_{M} \zeta_{M}^{+}
$$

of the fibrewise suspension of the fibrewise one-point compactification $\zeta_{M}^{+}$of the vector bundle $\zeta$. The inclusion of the (pointed) sphere bundle

$$
\zeta_{M}^{+} \hookrightarrow \Omega_{M} \Sigma_{M} \zeta_{M}^{+}
$$

by the James construction (in each fibre) gives, as the image of the Thom class of the oriented vector bundle $\zeta$, a fibrewise homology class $z$ which generates the loop homology as a polynomial ring over $H^{*}(M)$ :

$$
\mathbb{H}_{M}^{*}(*)=\left(\mathbb{Z}[u] /\left(u^{2}\right)\right) \otimes \mathbb{Z}[z], \quad \text { where } u \in H^{2 n+1}(M), z \in \mathbb{H}_{M}^{-2 n}(*) .
$$

More generally, we can look at the loop homology of $M=N \times S^{2 n+1}$ for any compact ENR $N$. By the same method we obtain

$$
\tilde{\mathbb{H}}_{N \times S^{2 n+1}}^{*}(X)=\tilde{\mathbb{H}}_{N}^{*}(X) \otimes \mathbb{H}_{S^{2 n+1}}^{*}(*) .
$$

Example 3.10. Let $M=\mathrm{U}(n)$, where $n \geqslant 1$, be the unitary group. Then the bundle $\mathcal{L} M \rightarrow M$ is trivial as a fibrewise Hopf space: $\mathcal{L} \mathrm{U}(n)=\mathrm{U}(n) \times \Omega \mathrm{U}(n)$. So we may write down the loop homology as

$$
\mathbb{H}_{\mathrm{U}(n)}^{*}(*)=H^{*}(M) \otimes \mathbb{Z}\left[x_{0}, x_{0}^{-1}\right]\left[x_{1}, \ldots, x_{n-1}\right],
$$

where $x_{i} \in \mathbb{H}_{M}^{-2 i}(*)$. (For the related calculation of the fibrewise Pontrjagin ring of the fibrewise Hopf space $\mathcal{L} B \mathrm{U}(n) \rightarrow B \mathrm{U}(n)$ with fibre $\mathrm{U}(n)$, see $[\mathbf{8}, \mathbf{9}]$.)

This includes the special case $M=\mathrm{U}(1)=S^{1}$, for which the free loop space $\mathcal{L} M \rightarrow M$ is fibre homotopy equivalent to $M \times \mathbb{Z} \rightarrow M$ and

$$
\mathbb{H}_{M}^{*}(*)=\left(\mathbb{Z}[u] /\left(u^{2}\right)\right) \otimes \mathbb{Z}\left[z, z^{-1}\right], \quad \text { where } u \in H^{1}(M), z \in \mathbb{H}_{M}^{0}(*) .
$$

In [2] we established a fibrewise stable splitting of $\mathcal{L} M \rightarrow M$ when $M$ is a sphere or real projective space. This result specializes to give the following additive decomposition of the loop homology.

Example 3.11. Let $M$ be the real projective space of dimension $d$. Then

$$
\mathbb{H}_{M}^{*}(*)=H^{*}(M) \oplus \bigoplus_{l \geqslant 1} \tilde{H}^{*+l}\left(S(\tau M)^{-l \pi^{*} \tau M}\right),
$$

as a module over $H^{*}(M)$, where $\pi: S(\tau M) \rightarrow M$ is the sphere bundle of the tangent bundle $\tau M$. (Here and elsewhere we write $B^{\xi}$ for the Thom space of a finite-dimensional real vector bundle $\xi$ over a base $B$.) 
The Serre spectral sequence in Proposition 3.8 was used by Cohen et al. [5] to calculate the loop homology of even- (and odd-) dimensional spheres and of complex projective spaces. (See also [9, Part II, Propositions 15.30 and 15.33] and [1] for similar calculations of fibrewise Pontrjagin rings.)

In considering manifolds with boundary, as in the next section, we shall require the following extension of the basic definition.

Definition 3.12. Let $A$ be a closed sub-ENR of the compact ENR $M$. Then we define

$$
\tilde{\mathbb{H}}_{(M, A)}^{*}(X)=H_{(M, A)}^{*}\left\{M \times X ;(\mathcal{L} M)_{+M}\right\} .
$$

The Pontrjagin product provides an associative loop multiplication

$$
\mu: \tilde{\mathbb{H}}_{(M, A)}^{*}(X) \otimes \tilde{\mathbb{H}}_{(M, A)}^{*}(Y) \rightarrow \tilde{\mathbb{H}}_{(M, A)}^{*}(X \wedge Y) .
$$

In particular, $\mathbb{H}_{(M, A)}^{*}(*)$ is an associative $H^{*}(M)$-algebra. It will not normally possess an identity element if $A$ is non-empty.

Example 3.13. Indeed, if $M$ is the disc $D^{d}, d \geqslant 1$, and $A$ is its boundary $\partial D^{d}$, the loop homology algebra is $\mathbb{Z}$ concentrated in dimension $d$. For the inclusion, $M \times S^{0} \rightarrow(\mathcal{L} M)_{+M}$ is a fibre homotopy equivalence and we may, more generally, calculate

$$
\tilde{\mathbb{H}}_{(M, A)}^{*}(X)=H_{(M, A)}^{*}\left\{M \times X ; M \times S^{0}\right\}=\tilde{H}^{*-d}(X) .
$$

\section{The loop homology of a smooth manifold}

We recall the following result from [10, 9 , particularly Lemmas 9.3 and 9.11].

Proposition 4.1. Suppose that $M$ is a closed (smooth) manifold, with tangent bundle $\tau M$. Then there is a natural equivalence,

$$
\lambda_{M}: H_{M}^{*}\{M \times X ; F\} \stackrel{\cong}{\rightrightarrows} H^{*}\left\{X ;(F, M)^{-p^{*} \tau M}\right\},
$$

for any finite pointed complex $X$ and any pointed homotopy fibre bundle $p: F \rightarrow M$ with fibres of the homotopy type of $C W$ complexes. Naturality in $X$ and $F$ has the obvious meaning; naturality in $M$ is to be understood as follows. Suppose that $f: M^{\prime} \rightarrow M$ is a (continuous) map from a closed manifold $M^{\prime}$, and write $p^{\prime}: F^{\prime} \rightarrow M^{\prime}$ for the pull-back of $F \rightarrow M$. Then there is a commutative diagram

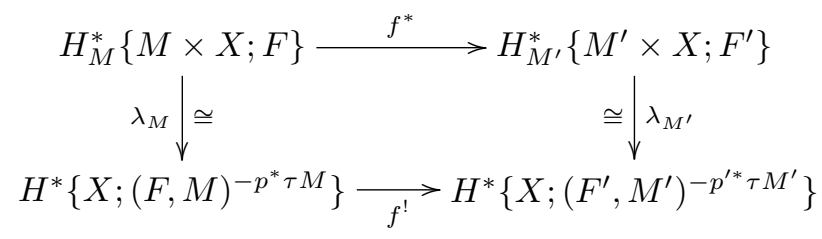

in which $f^{*}$ is given by lifting from $M$ to $M^{\prime}$ and the Umkehr map $f^{!}$is defined by the Pontrjagin-Thom construction. 
In the statement, the relative Thom space (or, rather, spectrum) $(F, M)^{-p^{*} \tau M}$ is the homotopy cofibre of the inclusion

$$
M^{-\tau M} \rightarrow F^{-p^{*} \tau M}
$$

of Thom spaces (given by the inclusion of the basepoint section $M \hookrightarrow F$ ).

Although the result in Proposition 4.1 was described in [10] as 'more or less standard', it was, perhaps, not so well known at that time. Other accounts have since become available (see, for example, [9, Part II, Corollary 12.41 and Proposition 12.43)] or [13]). When $F$ is a trivial bundle, the isomorphism $\lambda_{M}$ reduces to the Poincaré-Atiyah duality between $M_{+}$and $M^{-\tau M}$, and the naturality involving $f$ is the standard relation between the Umkehr $f^{!}$and the induced map $f^{*}$ under duality. As is explained in $[\mathbf{1 0}$, p. 401], the general result can be established, like the duality theorem, by constructing $\lambda_{M}$ and an inverse map $\mu_{M}$ explicitly using Umkehr maps and then checking, by an essentially formal argument using the properties of the Umkehr, that $\lambda_{M}$ and $\mu_{M}$ are mutual inverses. The correspondence between $f^{*}$ and $f^{!}$(which was not discussed in [10]) follows from the functoriality of the Umkehr map. It is also clear, from the nature of the proof, that the result (stated in [10] for stable cohomotopy) holds for other cohomology theories.

The correspondence in Proposition 4.1 allows us to relate our definition (Definition 3.3) of loop homology in terms of fibrewise homology to the definition given by Cohen and Jones in $[4]$.

Theorem 4.2. Let $M$ be a closed (smooth) manifold. Then there is a natural equivalence,

$$
\tilde{\mathbb{H}}_{M}^{*}(X)=H_{M}^{*}\left\{M \times X ;(\mathcal{L} M)_{+M}\right\} \stackrel{\lambda_{M}}{\longrightarrow} H^{*}\left\{X ;(\mathcal{L} M)^{-p^{*} \tau M}\right\},
$$

under which the fibrewise product in $\tilde{\mathbb{H}}_{M}^{*}(X)$ corresponds to the product defined by Cohen and Jones using the Pontrjagin-Thom construction.

Proof. We have to show that the fibrewise Pontrjagin product corresponds under the equivalence $\lambda_{M}$ of Proposition 4.1 to the Cohen-Jones version of the Chas-Sullivan multiplication. This will follow from the commutativity of the diagram in Figure 1, in which the left-hand column is a factorization of the loop multiplication $\mu$ and the righthand column defines the Cohen-Jones multiplication.

In the square [1], the maps $\wedge$ are given by the (exterior) smash product and $\mathcal{L} M \times \mathcal{L} M$ fibres over $M \times M$ by the projection $p \times p$. The map $\Delta$ in the square [2] is the diagonal $M \rightarrow M \times M$; commutativity follows from the naturality of the equivalence $\lambda$. The square [3] commutes by naturality of Proposition 4.1 in $F$.

When $M$ is an oriented closed manifold of dimension $d$, we may use the Thom isomorphism for $\tau M$ to identify $H^{*}\left\{X ;(\mathcal{L} M)^{-p^{*} \tau M}\right\}$ with the homology $H^{*-d}\left\{X ;(\mathcal{L} M)_{+}\right\}$of $\mathcal{L} M$ with a shift of degree $d$. This is the case considered by Chas and Sullivan in [3]. 


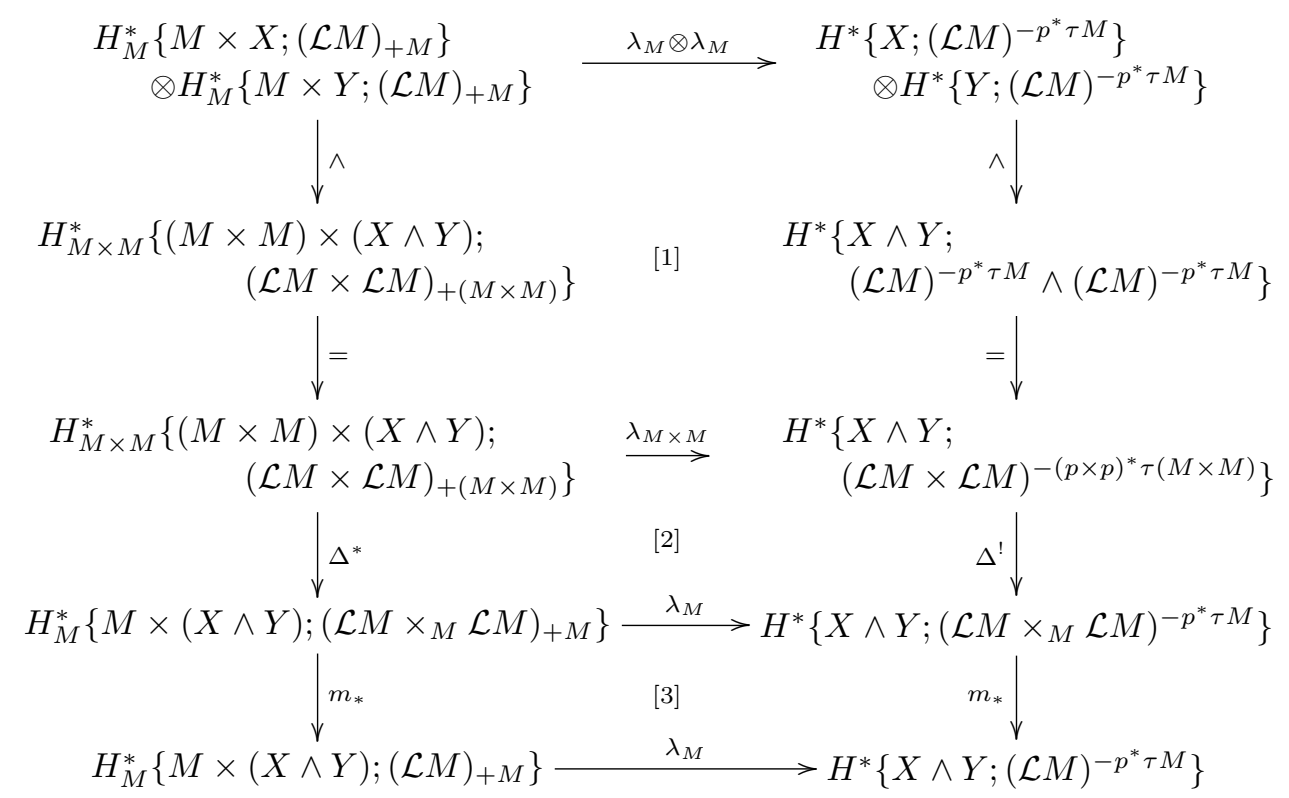

Figure 1.

The structure described in Propositions 3.4 and 3.5 translates under the equivalence in Theorem 4.2 into [4, Theorem 1(1) and (2)]. The Serre spectral sequence in Proposition 3.8 gives the spectral sequence constructed by an ad hoc method in [5]. The homotopy invariance (Theorem 3.7) leads, as we now explain, to the homotopy invariance of the Chas-Sullivan product established in [6]. Suppose that $f: M \rightarrow N$ is a homotopy equivalence between closed, smooth manifolds. Recall that there is an associated 'homotopy derivative' $\mathrm{d} f$, which is a stable fibre homotopy equivalence

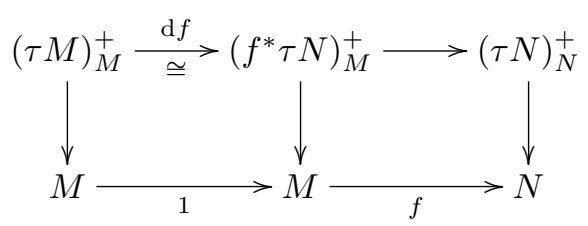

from the fibrewise one-point compactification of $\tau M$ over $M$ to that of the pull-back $f^{*} \tau N$ (see, for example, [7, Chapter 8]).

Proposition 4.3. Let $f: M \rightarrow N$ be a homotopy equivalence between closed, smooth manifolds. Then the equivalence $f_{\#}: \tilde{\mathbb{H}}_{M}^{*}(X) \rightarrow \tilde{\mathbb{H}}_{N}^{*}(X)$ (Theorem 3.7) of multiplicative cohomology theories corresponds under the isomorphism in Theorem 4.2 to the composition

$$
(f, \mathrm{~d} f)_{*}: H^{*}\left\{X ;(\mathcal{L} M)^{-p^{*} \tau M}\right\} \stackrel{(\mathrm{d} f)_{*}}{\cong} H^{*}\left\{X ;(\mathcal{L} M)^{-p^{*} f^{*} \tau N}\right\} \stackrel{f_{*}}{\cong} H^{*}\left\{X ;(\mathcal{L} N)^{-q^{*} \tau N}\right\},
$$

where $q$ is the projection $\mathcal{L} N \rightarrow N$. 
Proof. First, we have by the naturality of $\lambda$ a commutative diagram:

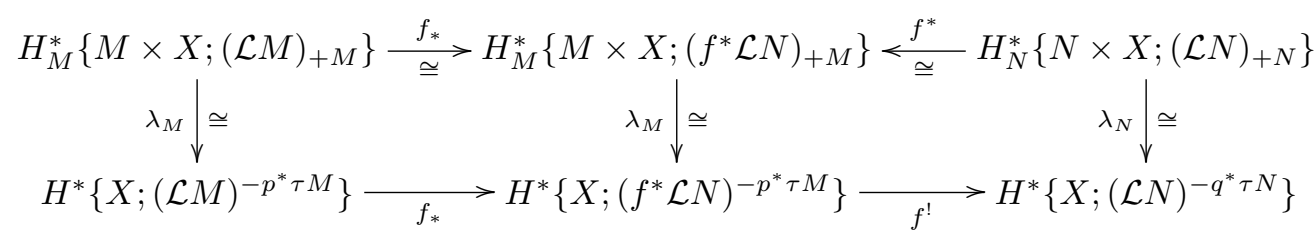

Since $\mathrm{d} f$ is a fibrewise equivalence over $M$, we may replace $f_{*} \circ(\mathrm{d} f)_{*}$ in the square

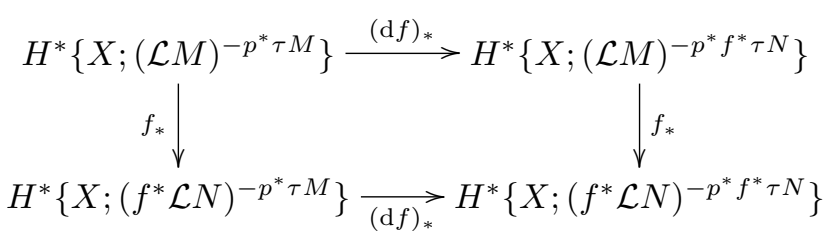

by $(\mathrm{d} f)_{*} \circ f_{*}$. It remains, therefore, to show that the inverse of the Umkehr map $f^{!}$above is the composition

$$
H^{*}\left\{X ;\left(f^{*} \mathcal{L} N\right)^{-p^{*} \tau M}\right\} \stackrel{(\mathrm{d} f)_{*}}{\longrightarrow} H^{*}\left\{X ;\left(f^{*} \mathcal{L} N\right)^{-p^{*} f^{*} \tau N}\right\} \stackrel{f_{*}}{\longrightarrow} H^{*}\left\{X ;(\mathcal{L} N)^{-q^{*} \tau N}\right\} .
$$

But this is a standard result about the Umkehr map for a homotopy equivalence (following from the definition of the homotopy derivative itself in terms of the Umkehr map); see, for example, [7].

This proof of homotopy invariance is essentially that given by Gruher and Salvatore in $[\mathbf{1 2}]$, except that they do not use fibrewise homology and work entirely within the Cohen-Jones framework.

The equivalence in Proposition 4.1 extends to manifolds with boundary (see [9, Part II, Proposition 12.44]).

Proposition 4.4. Let $M$ be a compact manifold with boundary. Then there are natural equivalences

$$
H_{(M, \partial M)}^{*}\{M \times X ; F\}=H^{*}\left\{X ;(F, M)^{-p^{*} \tau M}\right\}
$$

and

$$
H_{M}^{*}\{M \times X ; F\}=H^{*}\left\{X ;(F,(F \mid \partial M) \cup M)^{-p^{*} \tau M}\right\}
$$

for a pointed finite complex $X$ and pointed homotopy fibre bundle $p: F \rightarrow M$ with fibres of the homotopy type of $C W$ complexes.

Specializing to $(\mathcal{L} M)_{+M} \rightarrow M$ we obtain the following.

Proposition 4.5. For a compact manifold $M$ with boundary,

$$
\tilde{\mathbb{H}}_{(M, \partial M)}^{*}(X)=H^{*}\left\{X ;(\mathcal{L} M)^{-p^{*} \tau M}\right\}
$$


and

$$
\tilde{\mathbb{H}}_{M}^{*}(X)=H^{*}\left\{X ;(\mathcal{L} M, \mathcal{L} M \mid \partial M)^{-p^{*} \tau M}\right\},
$$

where $\mathcal{L} M \mid \partial M$ is the space of free loops $\alpha: \mathbb{R} / \mathbb{Z} \rightarrow M$ with $\alpha(0) \in \partial M$ (that is, beginning on the boundary).

Example 4.6. Let $\Gamma$ be a finite subgroup of the group $\operatorname{Sp}(1)$ of unit quaternions and take $M=\operatorname{Sp}(1) / \Gamma$. For $\gamma \in \Gamma$, let $\Omega_{\gamma}$ denote the space of paths $\omega:[0,1] \rightarrow \operatorname{Sp}(1)$ from $1=\omega(0)$ to $\gamma=\omega(1)$; it is homotopy equivalent to $\Omega \mathrm{Sp}(1)$. Associated to the adjoint action of $\Gamma$ on itself by inner automorphisms there is the bundle of groups $\Phi=$ $\operatorname{Sp}(1) \times_{\Gamma} \Gamma \rightarrow \operatorname{Sp}(1) / \Gamma=M$. A loop $\alpha \in \mathcal{L} M$ based at $g \Gamma \in \operatorname{Sp}(1) / \Gamma$, where $g \in \operatorname{Sp}(1)$, lifts to a (unique) path $g \omega$, with $\omega \in \Omega_{\gamma}$, from $g$ to $g \gamma$ in $\operatorname{Sp}(1)$, and the assignment $\alpha \mapsto[g \Gamma, \gamma] \in \Phi$ defines a fibrewise Hopf map $\rho: \mathcal{L} M \rightarrow \Phi$. For both $\mathcal{L} M$ and $\Phi$ the path components correspond to the conjugacy classes $[\gamma]$ in $\Gamma$. The component $\mathcal{L}^{\gamma} M$ of $\mathcal{L} M$ corresponding to $[\gamma]$ is identified with $(\operatorname{Sp}(1) / C(\gamma)) \times \Omega_{\gamma}$, where $C(\gamma)$ is the centralizer of $\gamma$ in $\Gamma$, by mapping $\alpha$ in the description above to $(g C(\gamma), \omega)$. The $\gamma$-component $\Phi^{\gamma}$ of $\Phi$ is $\operatorname{Sp}(1) \times{ }_{\Gamma}[\gamma]=\operatorname{Sp}(1) / C(\gamma)$, and $\rho$ restricts to the map $\mathcal{L}^{\gamma} M \rightarrow \Phi^{\gamma}$ projecting onto the first factor.

Composition with a chosen element of $\Omega_{\gamma}$ gives a homotopy equivalence $\Omega S^{3}=\Omega_{1} \rightarrow$ $\Omega_{\gamma}$. Using Proposition 4.1 and then Poincaré duality for each component to make the identification

$$
H_{M}^{*}\left\{M \times S^{0} ;(\operatorname{Sp}(1) / C(\gamma))_{+M}\right\}=H^{*}(\operatorname{Sp}(1) / C(\gamma)),
$$

we obtain an additive decomposition of the loop homology as

$$
\mathbb{H}_{M}^{*}(*)=\bigoplus_{[\gamma]} H^{*}(\operatorname{Sp}(1) / C(\gamma)) \otimes \mathbb{Z}[z]
$$

where $z \in \mathbb{H}_{M}^{-2}(*)$ comes from a generator of $H_{-*}\left(\Omega S^{3}\right)=\mathbb{Z}[z]$.

The homology $H^{0}\left\{S^{0} ; \Omega(M, \Gamma)_{+}\right\}$of the fibre at $\Gamma \in M$ is the group ring $\mathbb{Z}[\Gamma]$. The loop homology $\mathbb{H}_{M}^{0}(*)=H_{M}^{0}\left\{M \times S^{0} ;(\mathcal{L} M)_{+M}\right\}$ in dimension 0 is identified by restriction to the fibre at $\Gamma$ with the (commutative) ring of invariants $\mathbb{Z}[\Gamma]^{\Gamma}$, which as a free abelian group has a basis indexed by the conjugacy classes of $\Gamma$. Moreover, the induced maps

$$
\rho_{*}: \mathbb{Z}[\Gamma]=H^{0}\left\{S^{0} ; \Omega(M, \Gamma)_{+}\right\} \rightarrow H^{0}\left\{S^{0} ; \Phi_{\Gamma}\right\}
$$

and

$$
\rho_{*}: \mathbb{Z}[\Gamma]^{\Gamma}=H_{M}^{0}\left\{M \times S^{0} ;(\mathcal{L} M)_{+M}\right\} \rightarrow H_{M}^{0}\left\{M \times S^{0} ; \Phi_{+M}\right\}
$$

are isomorphisms.

\section{Commutativity}

Of course, the fibrewise Hopf space $\mathcal{L} M=\Omega_{M}(M \times M) \rightarrow M$ is not normally homotopycommutative (if only because the fibre need not be homotopy-commutative) and the Pontrjagin ring $H_{M}^{*}\left\{E ;(\mathcal{L} M)_{+M}\right\}$ for a non-trivial fibrewise pointed space $E \rightarrow M$ need not be commutative (see [11] for discussion of a related commutativity problem). 
Example 5.1. In Example 4.6, suppose that the group $\Gamma$ is non-abelian. Then the ring $H_{M}^{0}\left\{\left(\Phi \times_{M} \Phi\right)_{+M} ;(\mathcal{L} M)_{+M}\right\}$ is not commutative. For it maps surjectively to $H_{M}^{0}\left\{\left(\Phi \times_{M}\right.\right.$ $\left.\Phi)_{+M} ; \Phi_{+M}\right\}$ by the ring homomorphism $\rho_{*}$, and this ring, which contains the Hopf multiplication of $\Phi$, cannot be commutative, because the homology $H^{0}\left\{S^{0} ; \Phi_{\Gamma}\right\}$ of a fibre is not.

But the loop homology ring of a closed manifold is commutative, as we shall verify below.

Proposition 5.2. Let $M$ be a closed smooth manifold. Then the loop product $\mu$ is commutative, that is, for pointed finite complexes $X$ and $Y$ there is a commutative diagram:

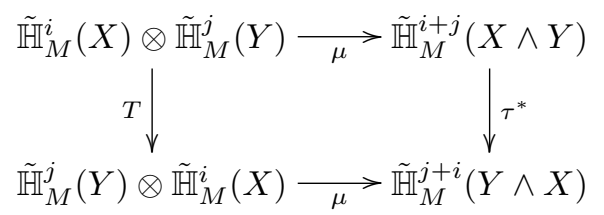

where $\tau: X \wedge Y \rightarrow Y \wedge X$ is the canonical equivalence and $T$ is the algebraic transposition (including the sign). In particular, $\mathbb{H}_{M}^{*}(*)$ is a (super) commutative graded algebra over $H^{*}(M)$.

The commutativity of the ring $\mathbb{H}_{M}^{*}(*)$ when $M$ is orientable was demonstrated by Chas and Sullivan in [3]. The proof below derives from ideas of Cohen and Jones which were included in a preprint draft of [4] but did not appear in the published version. Another, rather different, proof is given by Klein in [14, p. 1826]; see Remark 5.10.

Suppose, more generally, that $M$ is a compact manifold, possibly with non-empty boundary, of dimension $d$.

For a loop $\alpha: \mathbb{R} / \mathbb{Z} \rightarrow M$ and $t \in \mathbb{R}$, we shall abbreviate $\alpha(t+\mathbb{Z})$ to $\alpha(t)$. Consider the mapping

$$
q: \mathcal{L} M \times \mathbb{R} \rightarrow M, \quad(\alpha, t) \mapsto \alpha(t) .
$$

Since the space $\mathcal{L} M$ is metrizable, it is paracompact, and there is a vector bundle isomorphism

$$
\theta: p^{*} \tau M \rightarrow q^{*} \tau M \quad(\text { over } \mathcal{L} M \times \mathbb{R})
$$

that restricts to the identity on $M \times\{0\}$. (To be precise, we should write $p^{*} \tau M \times\{0\}$ instead of simply $p^{*} \tau M$.) Moreover, such an isomorphism $\theta$ is unique up to homotopy. It determines, for each loop $\alpha \in \mathcal{L} M$, a family of vector space isomorphisms

$$
\theta(\alpha, t): \tau_{\alpha(0)} M \rightarrow \tau_{\alpha(t)} M, \quad t \in \mathbb{R},
$$

with $\theta(\alpha, 0)=1$.

Translation $\theta(\alpha, 1): T_{\alpha(0)} M \rightarrow T_{\alpha(0)} M$ once around a loop yields a homotopy class $h \in\left[(\mathcal{L} M)_{+} ; \mathrm{O}(2 d+1)\right]$, which is independent of the choice of the isomorphism $\theta$ (because any two are homotopic). For we can embed $\tau M$, uniquely up to homotopy, in a trivial bundle $M \times \mathbb{R}^{2 d+1}$ over $M$ and define $h(\alpha)$ to be $\theta(\alpha, 1) \oplus 1: \mathbb{R}^{2 d+1} \rightarrow \mathbb{R}^{2 d+1}$. 
Remark 5.3. If the space $\mathcal{L} M$ of continuous loops is replaced by the space of smooth loops, we can specify an isomorphism $\theta$ by choosing a (smooth) Riemannian metric on $M$. Parallel translation with respect to the Riemannian connection along any closed smooth loop $\alpha$ then gives an isomorphism $\theta(\alpha, t): \tau_{\alpha(0)} M \rightarrow \tau_{\alpha(t)} M$. The corresponding class $h$ is the (stable) holonomy.

The holonomy map $h$ determines (by the fibrewise version of the classical $J$-construction) a self-map

$$
h_{*}: \Sigma_{M}^{2 d+1}(\mathcal{L} M)_{+M} \rightarrow \Sigma_{M}^{2 d+1}(\mathcal{L} M)_{+M}
$$

of the fibrewise suspension of $(\mathcal{L} M)_{+M}$ over $M$. We shall show in Lemma 5.4 that $h_{*}$ acts as the identity on loop homology.

The proof exploits the action of the group $\mathbb{R}$ on $\mathcal{L} M$ by rotating loops:

$$
(t \cdot \alpha)(u)=\alpha(t+u), \quad t, u \in \mathbb{R} .
$$

Using the map $\theta$ we can lift rotation through $t \in \mathbb{R}$ to an automorphism $f_{t}$ of the vector bundle $p^{*} \tau M$ over $\mathcal{L} M$ :

$$
(\alpha, v) \mapsto(t \cdot \alpha, \theta(\alpha, t) v), \quad v \in \tau_{\alpha(0)} M,
$$

and this vector bundle automorphism induces a self-map

$$
\left(f_{t}\right)_{*}:(\mathcal{L} M)^{-p^{*} \tau M} \rightarrow(\mathcal{L} M)^{-p^{*} \tau M}
$$

of the Thom space of the virtual bundle $-p^{*} \tau M$. (A discussion of the category of virtual bundles can be found, for example, in [9, Part II, $\S 8)]$.) Here is a concrete description of $\left(f_{t}\right)_{*}$. Let $\nu$ be the normal bundle of an embedding of $M$ in $\mathbb{R}^{2 d+1}$, so that $\tau M \oplus \nu=\mathbb{R}^{2 d+1}$ is trivial. Then $(\mathcal{L} M)^{-p^{*} \tau M}$ is the $(2 d+1)$-fold desuspension of the Thom space of $p^{*} \nu$. Moreover, there is a vector bundle isomorphism $\phi: p^{*} \nu \rightarrow q^{*} \nu$ over $\mathcal{L} M \times \mathbb{R}$ restricting to the identity on $\mathcal{L} M \times\{0\}$, and the sum $\theta \oplus \phi$ is homotopic to the identity automorphism of the trivial bundle $\mathbb{R}^{2 d+1}$ over $\mathcal{L} M \times \mathbb{R}$. For $t \in \mathbb{R}$, let $g_{t}$ be the vector bundle automorphism

$$
(\alpha, w) \mapsto(t \cdot \alpha, \phi(\alpha, t) w), \quad w \in \nu_{\alpha(0)},
$$

of $p^{*} \nu$. Then $\left(f_{t}\right)_{*}$ is the $(2 d+1)$-fold desuspension of $\left(g_{t}\right)_{*}$.

Lemma 5.4. Composition with the map $h_{*}$, (5.1), given by the holonomy $h \in$ $\left[(\mathcal{L} M)_{+} ; \mathrm{O}(2 d+1)\right]$ induces the identity on

$$
\tilde{\mathbb{H}}_{(M, \partial M)}^{*}(Z)=H_{(M, \partial M)}^{*}\left\{M \times Z ;(\mathcal{L} M)_{+M}\right\}
$$

for any pointed finite complex $Z$.

Proof. The proof depends upon the following elementary observation. Let $\xi$ be a finite-dimensional real vector bundle over a space $B$. Suppose that $a$ and $b$ are automorphisms, over $B$, of the trivial bundle $B \times \mathbb{R}^{n}$ and of $\xi$, respectively, such that $a \oplus 1$ 
and $1 \oplus b$ are homotopic as automorphisms of the direct sum $\mathbb{R}^{n} \oplus \xi$. Then the induced self-maps of the Thom space of $\mathbb{R}^{n} \oplus \xi$ are homotopic:

$$
(a \oplus 1)_{*} \simeq 1 \wedge b_{*}: B^{\mathbb{R}^{n} \oplus \xi}=\Sigma^{n}\left(B^{\xi}\right) \rightarrow B^{\mathbb{R}^{n} \oplus \xi}=\Sigma^{n}\left(B^{\xi}\right) .
$$

The map $\left(f_{1}\right)_{*}$, being homotopic to $\left(f_{0}\right)_{*}=1$, induces the identity on the homology $H^{*}\left\{Z ;(\mathcal{L} M)^{-p^{*} \tau M}\right\}$ of the Thom space. But $f_{1}$ is a fibre-preserving map over $\mathcal{L} M$. We now apply the observation above (with $\xi$ the pull-back $p^{*} \nu$ of the normal bundle $\nu$ to $B=\mathcal{L} M, b=g_{1}$ and $\left.a=h^{-1}\right)$ to see that $\left(f_{1}\right)_{*}$ is induced by $h^{-1}$, regarded as an automorphism of the trivial bundle $\mathcal{L} M \times \mathbb{R}^{2 d+1}$ over $\mathcal{L} M$. Hence, $h^{-1}$, and so also $h$, induce the identity on $H^{*}\left\{Z ;(\mathcal{L} M)^{-p^{*} \tau M}\right\}$.

Under the equivalence in Theorem 4.2, the action of $h$ on $(\mathcal{L} M)^{-\tau M}$ corresponds to composition with the self-map $h_{*}$ in (5.1).

Remark 5.5. The class $h$ is not, in general, trivial. Indeed, if $M$ is not orientable, then the restriction to a single loop $\alpha \in \mathcal{L} M$ may be non-trivial. In homology $h$ gives a class in $H^{0}(\mathcal{L} M)$ which will be \pm 1 on a component: +1 on the component $\mathcal{L}^{\alpha} M$ of a loop $\alpha$ such that the bundle $\alpha^{*} \tau M$ is orientable (so trivial), -1 where the bundle is non-orientable.

It follows from Lemma 5.4 that for the component $\mathcal{L}^{\alpha} M$ of a loop $\alpha$ such that $\alpha^{*} \tau M$ is non-orientable,

$$
2 H_{(M, \partial M)}^{*}\left\{M \times Z ;\left(\mathcal{L}^{\alpha} M\right)_{+M}\right\}=0 .
$$

The standard loop multiplication $\mu: \mathcal{L} M \times_{M} \mathcal{L} M \rightarrow \mathcal{L} M$, taking an ordered pair $(\alpha, \beta)$ with $\alpha(0)=\beta(0)$ to the loop $\gamma$ defined by

$$
\gamma(t)= \begin{cases}\alpha(2 t) & \text { if } 0 \leqslant t \leqslant \frac{1}{2} \\ \beta(2 t-1) & \text { if } \frac{1}{2} \leqslant t \leqslant 1\end{cases}
$$

identifies the fibre product with the subspace

$$
\mathcal{L}_{2} M=\left\{\gamma \in \mathcal{L} M \mid \gamma(0)=\gamma\left(\frac{1}{2}\right)\right\}
$$

of $\mathcal{L} M$. Rotation by $\frac{1}{2} \in \mathbb{R}$ preserves the subspace $\mathcal{L}_{2} M$ and $f_{1 / 2}$ corresponds on the fibre product to the map induced by the automorphism

$$
(\alpha, \beta, v) \mapsto\left(\beta, \alpha, \theta\left(\alpha, \frac{1}{2}\right) v\right), \quad v \in \tau_{\alpha(0)=\beta(0)} M,
$$

of the bundle $p^{*} \tau M$ over $\mathcal{L} M \times_{M} \mathcal{L} M$.

Lemma 5.6. Let $t: \mathcal{L} M \times_{M} \mathcal{L} M \rightarrow \mathcal{L} M \times_{M} \mathcal{L} M$ be the map that interchanges the two factors and let $h_{*}$ be as in (5.1). Then $\mu_{*} \circ t \circ\left(1 \wedge h_{*}\right)=\mu_{*}$ :

$$
H_{(M, \partial M)}^{*}\left\{M \times Z ;\left(\mathcal{L} M \times_{M} \mathcal{L} M\right)_{+M}\right\} \rightarrow H_{(M, \partial M)}^{*}\left\{M \times Z ;(\mathcal{L} M)_{+M}\right\} .
$$


Proof. This is similar to the proof of Lemma 5.4. On $(\mathcal{L} M)^{-p^{*} \tau M}$ the map $\left(f_{1 / 2}\right)_{*}$ is homotopic to 1 , and so induces the identity on $H^{*}\left\{Z ;(\mathcal{L} M)^{-\tau M}\right\}$. Over the subspace $\mathcal{L}_{2} M$, the map $f_{1 / 2}$ is fibre preserving, and the description above shows that $\left(f_{1 / 2}\right)_{*}$ on $\left(\mathcal{L}_{2} M\right)^{-p^{*} \tau M}$ is induced by $t \circ\left(h^{-1} \wedge 1\right)$. It follows that $\mu_{*} \circ t \circ\left(h_{*}^{-1} \wedge 1\right)=\mu_{*}$. The rest is algebra. By squaring, it is found that $\mu_{*}=\mu_{*} \circ\left(h_{*}^{-1} \wedge h_{*}^{-1}\right)$. Multiplying by $h_{*} \wedge h_{*}$ then yields the result.

With this preparation, we are ready to prove the following extension of Proposition 5.2 to manifolds with boundary.

Theorem 5.7 (commutativity of the loop product). Let $M$ be a compact smooth manifold. Then the loop multiplication $\mu$ is commutative, that is, the square

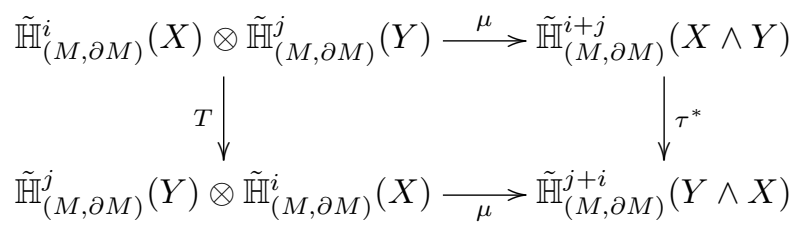

is commutative for pointed finite complexes $X$ and $Y$.

Proof. The multiplication $\mu$ factors as

$$
\begin{aligned}
H_{(M, \partial M)}^{*}\left\{M \times X ;(\mathcal{L} M)_{+}\right. & \otimes H_{(M, \partial M)}^{*}\left\{M \times Y ;(\mathcal{L} M)_{+M}\right\} \\
& \stackrel{\wedge}{\rightarrow} H_{(M, \partial M)}^{*}\left\{M \times(X \wedge Y) ;\left(\mathcal{L} M \times_{M} \mathcal{L} M\right)_{+M}\right\} \\
& \stackrel{\mu_{*}}{\longrightarrow} H_{(M, \partial M)}^{*}\left\{M \times(X \wedge Y) ;(\mathcal{L} M)_{+M}\right\} .
\end{aligned}
$$

Let $x \in \tilde{\mathbb{H}}_{(M, \partial M)}^{i}(X), y \in \tilde{\mathbb{H}}_{(M, \partial M)}^{j}(Y)$. From Lemma 5.6 with $Z=X \wedge Y$, we have

$$
x \cdot y=\mu_{*}(x \wedge y)=\mu_{*}\left(t\left(x \wedge h_{*} y\right)\right)=(-1)^{i j} \mu_{*}\left(h_{*} y \wedge x\right) .=(-1)^{i j} h_{*} y \cdot x .
$$

But $h_{*} y=y$, by Lemma 5.4 .

Remark 5.8. This argument will not apply to $\mathbb{H}_{M}^{*}(*)$ if the boundary is non-empty (because the rotation of loops in $\mathcal{L} M$ does not preserve the subspace $\mathcal{L} M \mid \partial M$ of loops $\alpha$ with $\alpha(0) \in \partial M)$, and the author does not know whether this ring, called the loop homology in this paper, is commutative in general. It may be that the term 'loop homology' is better reserved only for the groups $\mathbb{H}_{(M, \partial M)}^{*}(X)$, defined for a compact manifold $M$ of dimension $d$. When $M$ is orientable we have a Thom isomorphism

$$
\mathbb{H}_{(M, \partial M)}^{*}(*) \cong H_{d-*}(\mathcal{L} M),
$$

as in the original definition of Chas and Sullivan when $M$ is closed. This isomorphism lends support to the case for restricting the terminology. 
Remark 5.9. The proof of commutativity is much simpler if the manifold $M$ is orientable. In that case there is no need to look at the holonomy map $h$. For an orientation of $M$ determines a homological trivialization of $\tau M$ and so of the pull-back $p^{*} \tau M$ to $\mathcal{L} M$.

Remark 5.10. Klein's proof of commutativity in [14] depends on a different approach to the loop product. Suppose that $\phi: M \rightarrow B$ is a map to, say, a finite complex $B$, and let $\mathcal{M}$ be the space of pairs $(x, \alpha)$, where $x \in M$ and $\alpha:[0,1] \rightarrow B$ is a path in $B$ such that $\alpha(1)=\phi(x)$. Thus, we have a path-space fibration $\mathcal{M} \rightarrow B$, mapping $(x, \alpha)$ to $\alpha(0)$ and a homotopy equivalence $M \rightarrow \mathcal{M}$. Klein constructs, in the language of this paper, a composition product

$$
H_{M}^{*}\left\{M \times X ;\left(\phi^{*} \mathcal{M}\right)_{+M}\right\} \otimes H_{M}^{*}\left\{M \times Y ;\left(\phi^{*} \mathcal{M}\right)_{+M}\right\} \rightarrow H_{M}^{*}\left\{M \times(X \wedge Y) ;\left(\phi^{*} \mathcal{M}\right)_{+M}\right\}
$$

in the following way. Consider first the special case in which $M \rightarrow B$ is a fibre bundle. Replacing $\mathcal{M}$ by $M$, we may then make the obvious identification

$$
H_{M}^{*}\left\{M \times X ;\left(\phi^{*} M\right)_{+M}\right\}=H_{M}^{*}\left\{M \times X ;\left(M \times_{B} M\right)_{+M}\right\} \stackrel{\cong}{\rightarrow} H_{B}^{*}\left\{(B \times X) \wedge_{B} M_{+B} ; M_{+B}\right\} .
$$

The composition (see $\S 2.4$ ) defines an $H^{*}(B)$-bilinear product

$$
\begin{aligned}
H_{B}^{*}\left\{(B \times X) \wedge_{B} M_{+B} ; M_{+B}\right\} \otimes H_{B}^{*}\left\{(B \times Y) \wedge_{B}\right. & \left.M_{+B} ; M_{+B}\right\} \\
& \rightarrow H_{B}^{*}\left\{(B \times(X \wedge Y)) \wedge_{B} M_{+B} ; M_{+B}\right\},
\end{aligned}
$$

and this lifts to the required product on the $H_{M}^{*}$-groups above. In general, it is necessary to work with $\mathcal{M}$ rather than $M$ (which takes us outside the setting of $\S 2$ ).

When $\phi$ is the diagonal inclusion $M \rightarrow M \times M$, we may identify $\phi^{*} M \rightarrow M$ with the projection $p: \mathcal{L} M \rightarrow M$ and thus obtain a product on the loop homology.

This construction of the composition product works for any compact ENR $M$. If $M$ is a closed manifold, we may use $\lambda_{M}$ to make the identification

$$
H_{M}^{*}\left\{M \times X ;\left(\phi^{*} \mathcal{M}\right)_{+M}\right\} \stackrel{\cong}{\rightrightarrows} H^{*}\left\{X ;\left(\phi^{*} \mathcal{M}\right)^{-p^{*} \tau M}\right\} .
$$

In [14], this equivalence, in a rather more general setting, is called the 'norm map'.

\section{Other cohomology theories}

We have restricted our attention to cohomology with integer coefficients. Rational or mod 2 cohomology can be treated in exactly the same way. As Cohen and Jones observed in [4], one may just as easily replace ordinary cohomology by other cohomology theories, such as stable cohomotopy or $K$-theory (periodic or connective). (A brief introduction to fibrewise $K$-theory can be found in [9, Part II, $\S 15]$.) In the definition of the generalized loop homology and in establishing the main properties there is little to change, except that Remark 5.5 must be replaced by Remark 6.1, below. Otherwise, it is the only the specific computations performed in integral cohomology that may not be feasible when working with another cohomology theory. 
Remark 6.1. Let $\omega^{*}$, as in $[\mathbf{9}]$, denote stable cohomotopy. In the setting of Remark 5.5, consider the component $\mathcal{L}^{\alpha} M$ of a loop $\alpha$ such that $\alpha^{*} \tau M$ is non-trivial. Then

$$
2^{e} \omega_{(M, \partial M)}^{*}\left\{M \times Z ;\left(\mathcal{L}^{\alpha} M\right)_{+M}\right\}=0,
$$

where the exponent $e$ depends only on the dimension $d$ of $M$.

For the action of the holonomy $h_{*}$ in (5.1) lifts from a class in the group of units in the stable cohomotopy ring $\omega^{0}\left(\mathrm{O}^{-}(2 d+1)\right)$ of the orientation-reversing component of $\mathrm{O}(2 d+1)$, and this class has the form $-1+x$, where $x$ is nilpotent. If $x^{e}=0$, then $\left(2^{e-1}-2^{e-2} x+\cdots+(-1)^{e-1} x^{e-1}\right)(2-x)=2^{e}$.

Acknowledgements. I am grateful to the referee for explaining the relation between Klein's construction in [14] and the fibrewise theory and for drawing my attention to [12], which appeared at about the same time as this paper was submitted.

\section{References}

1. S. Bauer, M. C. Crabb and M. Spreafico, The classifying space of the gauge group of an SO(3)-bundle over $S^{2}$, Proc. R. Soc. Edinb. A 131 (2001), 767-783.

2. S. Bauer, M. C. Crabb and M. Spreafico, The space of free loops on a real projective space, in Groups of homotopy self-equivalences and related topics (ed. K. Maruyama and J. W. Rutter), Contemporary Mathematics, Volume 274, pp. 33-38 (American Mathematical Society, Providence, RI, 2001).

3. M. Chas And D. Sullivan, String topology, preprint math/GT/9911159.

4. R. L. Cohen AND J. D. S. Jones, A homotopy theoretic realization of string topology, Math. Annln 324 (2002), 773-798.

5. R. L. Cohen, J. D. S. Jones and J. Yan, The loop homology algebra of spheres and projective spaces, in Proc. Int. Conf. Categorical Decomposition Techniques in Algebraic Topology, Isle of Skye, 2001, Progress in Mathematics, Volume 215, pp. 72-92 (Birkhäuser, Basel, 2004).

6. R. L. Cohen, J. Klein and D. Sullivan, The homotopy invariance of the string topology loop product and string bracket, preprint math/GT/0509667.

7. M. C. CRABB, $\mathbb{Z} / 2$-homotopy theory (Cambridge University Press, 1980).

8. M. C. CrabB, Fibrewise homology, Glasgow Math. J. 43 (2001), 199-208.

9. M. C. Crabb and I. M. James, Fibrewise homotopy theory (Springer, 1998).

10. M. C. Crabb and W. A. Sutherland, The space of sections of a sphere-bundle, I, Proc. Edinb. Math. Soc. 29 (1986), 383-403.

11. M. C. Crabb And W. A. Sutherland, How non-abelian is non-abelian gauge theory?, Q. J. Math. 46 (1995), 279-290.

12. K. Gruher and P. Salvatore, Generalized string topology operations, preprint math/AT/0602210.

13. P. Hu, Higher string topology on general spaces, Proc. Lond. Math. Soc. 93 (2006), 515544 .

14. J. R. KLein, Fiber products, Poincaré duality and $A_{\infty}$-ring spectra, Proc. Am. Math. Soc. 134 (2006), 1825-1833.

15. S. A. Merkulov, De Rham model for string topology, Int. Math. Res. Not. 55 (2004), $2955-2981$.

16. C. ÖzEL, On smooth Chas-Sullivan loop product in Quillen's geometric complex cobordism of Hilbert manifolds, Topology Applicat. 153 (2006), 2104-2117. 